\title{
Correction to: Vedolizumab and Extraintestinal Manifestations in Inflammatory Bowel Disease
}

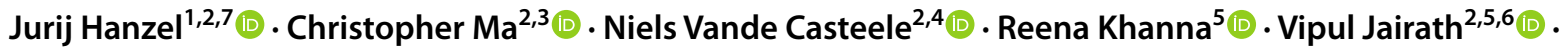 \\ Brian G. Feagan $2,5,6,8$ (D)
}

Published online: 28 August 2021

(c) Springer Nature Switzerland AG 2021

\section{Correction to: Drugs (2021) 81:333-347 \\ https://doi.org/10.1007/s40265-020-01460-3}

The third author whose name is currently presented as

Casteele N.V.

Should read

Vande Casteele N.

The original article can be found online at https://doi.org/10.1007/ s40265-020-01460-3.

Brian G. Feagan

brian.feagan@alimentiv.com

Jurij Hanzel

jurij.hanzel@alimentiv.com

Christopher Ma

christopher.ma@ucalgary.ca

Niels Vande Casteele

nvandecasteele@ucsd.edu

Reena Khanna

rkhanna3@uwo.ca

Vipul Jairath

vjairath@uwo.ca

1 Department of Gastroenterology, University Medical Center Ljubljana, Ljubljana, Slovenia

2 Alimentiv, \#200, 100 Dundas Street, London N6A 5B6, ON, Canada
3 Division of Gastroenterology and Hepatology, Cumming School of Medicine, University of Calgary, 6D61 Teaching Research Wellness Building, 3280 Hospital Drive NW, Calgary, Alberta T2N 4Z6, Canada

4 Department of Medicine, University of California San Diego, 9500 Gilman Drive \#0956, La Jolla, CA 92093, USA

5 Division of Gastroenterology, University of Western Ontario, 1151 Richmond Street, London N6A 2K7, ON, Canada

6 Department of Epidemiology and Biostatistics, University of Western Ontario, London, ON, Canada

7 Hullenbergweg 278-308, 1101 BV Amsterdam, The Netherlands

8 Department of Medicine, University of Western Ontario, London, ON, Canada 